\title{
Forensic Science Analyses of Cultural Heritage Objects
}

\author{
M. Kotrlý,* I. Turková,* and V.Grunwaldová ** \\ * Institute of Criminalistics Prague (ICP), POBox 62/KUP, Strojnicka 27, 17089 Praha 7, \\ Czech \\ Republic \\ ** Zentiva, a.s. Praha, U Kabelovny 130, 10237 Praha 10, Czech Republic
}

Forensic analysis of cultural heritage objects, or other works of art, constitutes a very wide and diverse area. The list of object types that we encounter in practice could be very extensive; several examples can be presented: certification of authenticity of works of art, namely paintings, attempted export of registered artistic monuments without appropriate licences, which are claimed to be worthless copies, covered by repaints, or otherwise altered in order to conceal their true identity, gemology frauds, etc. [1]

Recently examinations of paintings and gemology objects are the most frequent. In order to determine the authenticity of paintings, standard material analysis of colour layers is performed; its representative performance generally entails micro samples collection. This action is preceded by detailed documentation and screening in visible, UV and IR spectra and $\mathrm{X}$-ray imaging that according to our experience in some cases can provide more information than regular screening in IR area. Mobile XRF device proved useful for the choice of areas for micro samples collection. Although its current models cannot replace classic microanalysis, in most cases it enables type identification of possible pigment phases. The main limit of this method is the range of detectable elements. Principally it is possible to detect elements with atomic number higher than 14, which makes it impossible to identify pigments on the basis of light elements, like ultramarine blue. Another limit is given by a so-called matrix effect which occurs during direct work with surfaces of paintings. [2] The stratigraphy of the painting and radiation penetrating to various depths causes the loss of correct correlation between the content of the element detected and the intensity of the pulses. However, the intensity of the signal can be used for relative comparison of the points being measured that have similar base and stratigraphy. Classic embedded polished sections or microtome sections are prepared from collected micro samples. Microtome sections bring the advantage of optical microscopy, SEM EDS/WDS micro analysis, XRD micro diffraction and FTIR in transmission mode in a non-destructive manner and from a single and thus identical point of the sample, without its further division. Producing high-quality micro sections of a colour layer is not a technically banal matter which requires a suitable primary embedding material and it takes an experienced worker to carry out the sections. The material of the microtome knife, or its edge, the angle of the section, as well as its speed etc. - these all have effect. A special zerobackground plate made of monocrystalline silicon cut in plane (100) was tested for the fixation of the final section. The advantage of this plate is the possibility of safe transfer of the microtome section between analytical devices and the performance of the above-mentioned analyses without the need of further adjustment to other standard carriers. However, before the microtome section analyses are performed it is necessary to check the completeness of the strata sequence, e.g. according to the residue of original material in the embedding material. Experiments that were carried out and practical experience show that the risk of not maintaining or overlooking important layers is relatively high. Usually it is necessary to prepare more sections and subsequently choose the most suitable ones for further analyses. Optical microscopy dealing with individual layers description provides information on microstructure, texture and in some cases also enables basic identification of pigments or binding media. Therefore it is used for the initial examination of collected fragments, polished sections or sections, where it provides basic information on an unknown sample. Electron microscopy (SEM) allows identification of the sample phases according to the morphology of the grains and signals of SE and BSE detectors, pores identification according to the morphology etc. 
During cross-section examination it also allows stratigraphy study. In most cases SEM is used in combination with electron microanalysis - EDS/WDS.

Image information obtained through optical and electron microscopy is further processed by means of image analysis. It is a program that enables objective measurement of distribution, size and morphological element parameters. In active mode this software usually makes it possible to scan images from various sources, save them into archives and manually or automatically measure them. Out of many parameters that can be monitored through image analysis for the purposes of description of colour layer particles the ones most important are namely the parameters capturing shape and size, or parameters of orientation of elongated particles within painting layers. Morphological parameters of maximum and minimum Feret's diameter proved optimal for the interpretation of particle size distribution. Circumference and area of the particle are parameters concerning both the shape and size of particles.

XRD microdiffraction is commonly used for phase analysis. It enables examination from the area of about 100 micrometers and allows identification of the similar-order sizes as microscopic methods. It can also be used for analysis of individual pigment grains in a colour layer. The device combines regular X-ray with special monocapillary and a very fast semiconductor detector. The total reflection of X-ray beam takes place in a monocapillary. A divergent beam becomes a quasi-parallel one. Apart from the collimation of the X-ray beam the monocapillary acts as an energetic filter and suppresses white radiation. A monocapillary is optimized for $\mathrm{Cu} \mathrm{K \alpha}$ radiation but can also be applied for other types of radiation with energy lower than $10 \mathrm{keV}$ like $\mathrm{Cr}, \mathrm{Fe}$ and $\mathrm{Co} \mathrm{K} \alpha$. Position-sensitive detector processes the incoming signal without the loss of resolution quality. [3] XRD is an indispensible technique principally in cases when there is a need to interpret phases indistinguishable by means of the chemical composition analysis, or with compounds.

A practical example is the case of fake paintings of a renown author from the thirties, Jan Zrzavy, as well as a contemporary highly appreciated author Kristian Kodet. The total of 60 Zrzavy fake paintings and 12 Kodet paintings were examined by complex methods. In this case especially microscopic and X-ray methods helped identify the fakes. The counterfeiter was so skilled that not even master Kodet was able to identify whether or not the paintings were his work.

Microscopic methods, electron EDS/WDS, or XRF microanalysis are critical for the analysis of gemology objects. In some cases also X-ray methods are used. Transmission detector for electron or X-ray beams is efficient for fake pearls analysis.

As practical examples we can present attempted customs fraud with Columbian emeralds, fake ground moldavites (tektites) in form of green glass which is chemically very hard to distinguish, Czech garnet frauds and fake pearls.

An interesting area is the analysis of counterfeits and alterations of historical documents and chronicles in order to acquire claim to inheritance or restitution claim, or noble descent proving with a view to attain higher social prestige. Here, apart from the above mentioned methods, also handwriting analysis methods are often applied.

As an example we can present the alteration of the entry in the parish register in Bernartice from 1685 - 1709 concerning the Andel family of Ronovec. It was meant to prove Czech reigning dynasty affiliation for contemporary progeny.

\section{References}

[1] I. Turkova, M. Kotrly, EMC 2008, 635, Springer, Berlin Heidelberg, 2008.

[2] E. Ciliberto, G. Spoto: Modern Analytical Methods in Art and Archaeology. WileyInterscience, New York, 2000.

[3] M. Kotrlý, V. Grunwaldová, I. Turková: Acta Crystallographica, Vol. 64, C598. 2008

[4] Acknowledgements - microanalytical methods at ICP were supported by projects

RN19961997008, RN19982000005, RN20012003007, RN20052005001, VD20062008B10, VD20072010B15, VG20102015065. 